\title{
Análise de Ilhas de Calor e Frescor Utilizando-Se de Processamento Digital de Imagens - Estudo de Caso Município de São Paulo/SP
}

\author{
Lucas Barbosa Cavalcante $^{1}$ (D), Heliofábio Barros Gomes ${ }^{2}$ (D), \\ Aline da Silva Inácio Cavalcante ${ }^{2}$ (D), Bárbara Cristiane Alcides da $\operatorname{Costa}^{3}$ (D), \\ Monaira Cristiane Alcides da $\operatorname{Costa}^{3}$ (D), Celiane Mendes da Silva ${ }^{3}$ (D), Rafaela Lisboa $\operatorname{Costa}^{2}$ (D), \\ Rodrigo Lins da Rocha Júnior ${ }^{3}$ (D), Felipe Souza dos Santos ${ }^{3}$ (D), \\ Fabrício Daniel dos Santos Silva ${ }^{2}$ (D), Talvanes Lins e Silva Júnior ${ }^{1}$ \\ ${ }^{1}$ Departamento de Engenharia Civil, Centro Universitário Tiradentes, Universidade Tiradentes, \\ AL, Brasil. \\ ${ }^{2}$ Instituto de Ciências Atmosféricas, Universidade Federal de Alagoas, Instituto de Ciências \\ Atmosféricas, Universidade Federal de Alagoas, Maceió AL, Brasil. \\ ${ }^{3}$ Programa de Pós-Graduação em Meteorologia, Instituto de Ciências Atmosféricas, \\ Universidade Federal de Alagoas, Maceió, AL, Brasil.
}

Recebido em: 29 de Junho de 2020 - Aceito em: 19 de Agosto de 2020

\begin{abstract}
Resumo
As cidades brasileiras vêm crescendo de forma desordenada e sofrendo conturbados processos antropogênicos, o que transforma de forma severa a atmosfera local, influenciando na qualidade de vida da sociedade. Esta pesquisa tem por finalidade a análise da variação de temperatura através do uso de sensoriamento remoto, buscando identificar ilhas de calor e frescor, sua origem e a variação espaço-temporal, tendo como área de estudo o município de São Paulo, uma grande cidade com densidade demográfica de 7.398,26 hab/ $\mathrm{km}^{2}$ e mais de 12.000 .000 de habitantes. Optou-se por utilizar de imagens do satélite Landsat 5 e da metodologia do algoritmo SEBAL para cálculo de temperatura da superfície. Os resultados encontrados evidenciam que a variação de temperatura da superfície vem sofrendo aumento com o passar dos anos, o município de São Paulo, a presença de grandes zonas industriais, o crescimento urbano desordenado, a presença de impermeabilização do solo através de revestimento asfáltico, são alguns dos fatores que contribuem para o surgimento de ilhas de calor. Também ficou evidenciada a presença de zonas de frescor, boa parte delas em áreas arborizadas, o que colabora para a redução da temperatura e melhoramento da condição de vida da população.
\end{abstract}

Palavras-chave: sensoriamento remoto, temperatura, código livre.

\section{Heat and Freshness Islands Analysis Using Digital Image Processing - Case Study City of São Paulo/SP}

\begin{abstract}
Brazilian cities have been growing in a disorderly manner and undergoing a disturbed anthropogenic process, which severely transforms the local atmosphere, influencing the quality of life of society. The purpose of this research is to analyze the temperature variation through the use of remote sensing, seeking to identify heat and freshness islands, their origins and the space-behavioral variation, having as study area the Municipality of São Paulo, a large city with population density of 7,398.26 inhabitants $/ \mathrm{km}^{2}$ and more than $12,000,000$ inhabitants. We opted to use Landsat 5 satellite images and SEBAL algorithm methodology for surface temperature calculation. The results show that the surface temperature variation has been increasing over the years, the city of São Paulo, the presence of large industrial zones, the disordered urban growth, the presence of soil sealing through asphalt coating, are some of the factors that contribute to the emergence of heat islands. It was also evidenced the presence of freshness zones, most of them in wooded areas, which contributes to reducing the temperature and improving the living conditions of the population.
\end{abstract}

Keywords: remote sensing, temperature, open source.

Autor de correspondência: Lucas Barbosa Cavalcante, cavalcantelb@gmail.com. 


\section{Introdução}

Nas últimas décadas as cidades brasileiras vêm crescendo de forma desordenada e sofrendo conturbados processos antropogênicos, ocasionando assim transformações severas na atmosfera local, influenciando diretamente na qualidade de vida dos citadinos. Os dosséis urbanos são compostos por diversos materiais com características específicas de capacidade calorífica, condutividade térmica e por consequência de difusividade térmica. Segundo Gartland (2010), em geral são confeccionados com substâncias escuras que absorvem mais a radiação eletromagnética emitida pelo Sol, além de serem estanques, dificultando a infiltração de água, reduzindo ainda mais a umidade. A falta de grandes áreas vegetadas em conjunto com a falta de planejamento urbano, influencia de forma direta em modificações do balanço de energia, que em conjunto com a poluição do ar, colaboram com a formação de ilhas de calor. A preocupação com a qualidade de vida em ambiente urbano vem sendo estudada continuamente, buscando técnicas que auxiliem na compreensão e mitigação dos problemas decorrentes do aquecimento urbano.

Uma das técnicas de detecção de temperatura de forma remota mais disseminadas no meio científico é o sensoriamento remoto, que se utiliza de radiação eletromagnética na faixa do infravermelho termal (acima de $3 \mu \mathrm{m}$ ), para estudar medidas de temperatura da superfície de uma determinada região. Jensen (2009), afirma que, se um objeto tem temperatura acima do zero absoluto, este emite radiação eletromagnética. Tendo como base tal afirmação, conclui-se que todas as paisagens do cotidiano urbano emitem energia, que pode ser interpretada pelos sensores abordo de satélites, e assim analisadas em forma de variação da temperatura, fator principal para detecção da formação de ilhas de calor. Segundo Gartland (2010), as ilhas de calor são formadas em áreas urbanas e suburbanas, sendo capazes de criar suas próprias brisas, seus efeitos são sentidos em toda a troposfera, sendo mais intenso na camada limite.

Diante do exposto, o objetivo desta pesquisa é analisar através do uso de imagens de sensoriamento remoto, a variação da temperatura na cidade de São Paulo, pesquisando suas origens e buscando entender sua variação espaço-temporal, para isso foi escolhida uma imagem referente ao ano de 1992 e outra de 2006.

\section{Materiais e Métodos}

\section{1. Área Urbana}

A área de estudo é definida pelo município de São Paulo, capital do Estado de São Paulo, localizado no Sudeste do Brasil, como apresentado na Fig. 1. É a cidade mais populosa de todo o hemisfério Sul, com uma população total estimada, segundo o Instituto Brasileiro de Geo- grafia e Estatística IBGE (2019), de 12.252.023 habitantes, em uma área territorial de $1.521,110 \mathrm{~km}^{2}$ IBGE (2019) gerando uma densidade demográfica de 8.054,66 $\mathrm{hab} / \mathrm{km}^{2}$ além de ser o principal centro financeiro da América do Sul.

A cidade de São Paulo está localizada nas bordas da Serra do Mar, sobre o Trópico de Capricórnio, entre as coordenadas geográficas de canto inferior esquerdo $46^{\circ}$ $54^{\prime} 40,462^{\prime \prime} \mathrm{O}$ de latitude e $24^{\circ} 03^{\prime} 55,911^{\prime \prime} \mathrm{S}$ de longitude e superior direito de $46^{\circ} 18^{\prime} 15,244^{\prime \prime} \mathrm{O}$ de latitude e $23^{\circ}$ $18^{\prime} 39,110^{\prime}$ ' S de longitude, tendo como referência datum SIRGAS2000. Limita-se ao Leste pelos municípios de Diadema, Ferraz de Vasconcelos, Itaquequecetuba, Mauá, Santo André, São Bernardo do Campo e São Caetano do Sul; a Norte pelos municípios de Caieiras, Guarulhos e Mariporã; a Oeste por Cajamar, Cotia, Embu, Embuguaçu, Itapecerica da Serra, Juquitiba, Osasco, Santana de Parnaíba, Taboão da Serra e Barueri; e ao Sul pelos municípios de Itanhaeim e São Vicente.

\subsection{Classificação climática}

A cidade de São Paulo apresenta temperaturas médias mensais em torno de $19,2^{\circ} \mathrm{C}$, com precipitação acumulada anual em torno de 1.441,0 mm, conforme Fig. 2, e umidade relativa do ar com valores médios anuais de $78,4 \%$. A classificação climática segundo Köppen e Geiger (1928), está classificada como Cwa (Clima subtropical úmido), caracterizando-se por ter inverno seco, com temperaturas inferiores a $18{ }^{\circ} \mathrm{C}$, no município de São Paulo esta estação ocorre entre os meses de junho e agosto, e verão quente com temperaturas superando os $22{ }^{\circ} \mathrm{C}$, ocorrendo entre os meses de dezembro e fevereiro

\subsection{Metodologia}

\subsubsection{Dados utilizados}

Para o desenvolvimento deste trabalho, foram utilizados os seguintes dados e softwares:

- Imagens do satélite LANDSAT-5, sensor TM, referentes à órbita 219 , ponto 76 e 77 correspondentes às passagens dos dias 26/09/1992 e 14/09/2006;

- Bases cartográficas digitais do IBGE, em formato shapefile, referentes ao Brasil, por município, e ao Estado de São Paulo com a divisão dos seus municípios;

- Imagem Topodata (Valeriano, 2005; Valeriano et al., 2009) adquiridas junto a Divisão de Sensoriamento Remoto (DSR), do Instituto Nacional de Pesquisas Espaciais (INPE), referentes as articulações: 23S48, 23S465 e 24S48;

- Imagem Geocover, adquiridas junto a National Aeronautics and Space Administration (NASA), referentes ao ano 2000;

- Software denominado Sistema de Processamento de Informações Georreferenciadas Spring (1996), desenvolvido pelo INPE, em sua versão 5.2.5. 

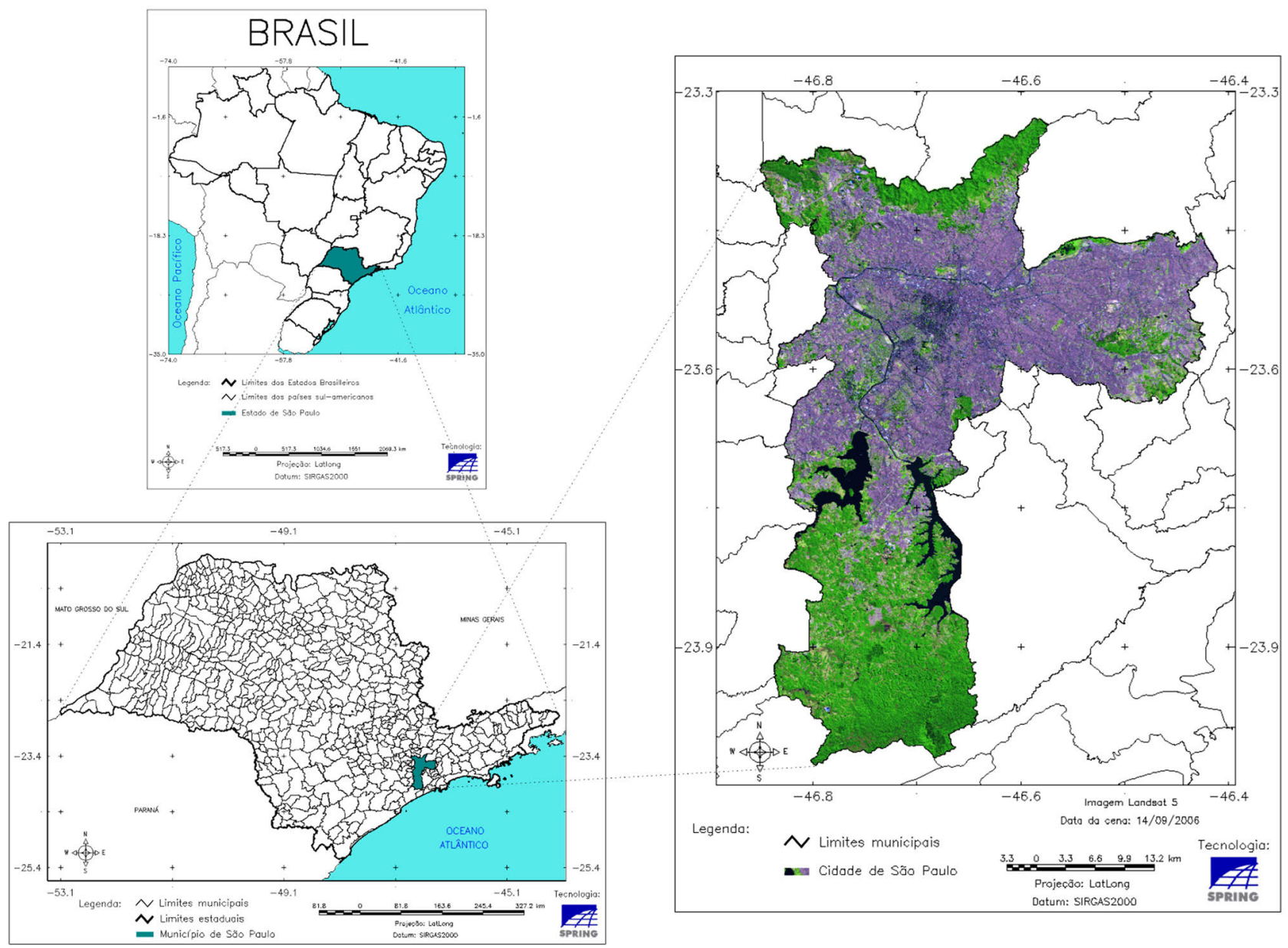

Figura 1 - Mapa de localização da área de estudo.

\section{Climograma - Cidade de São Paulo (Estação Mirante de Santana)}

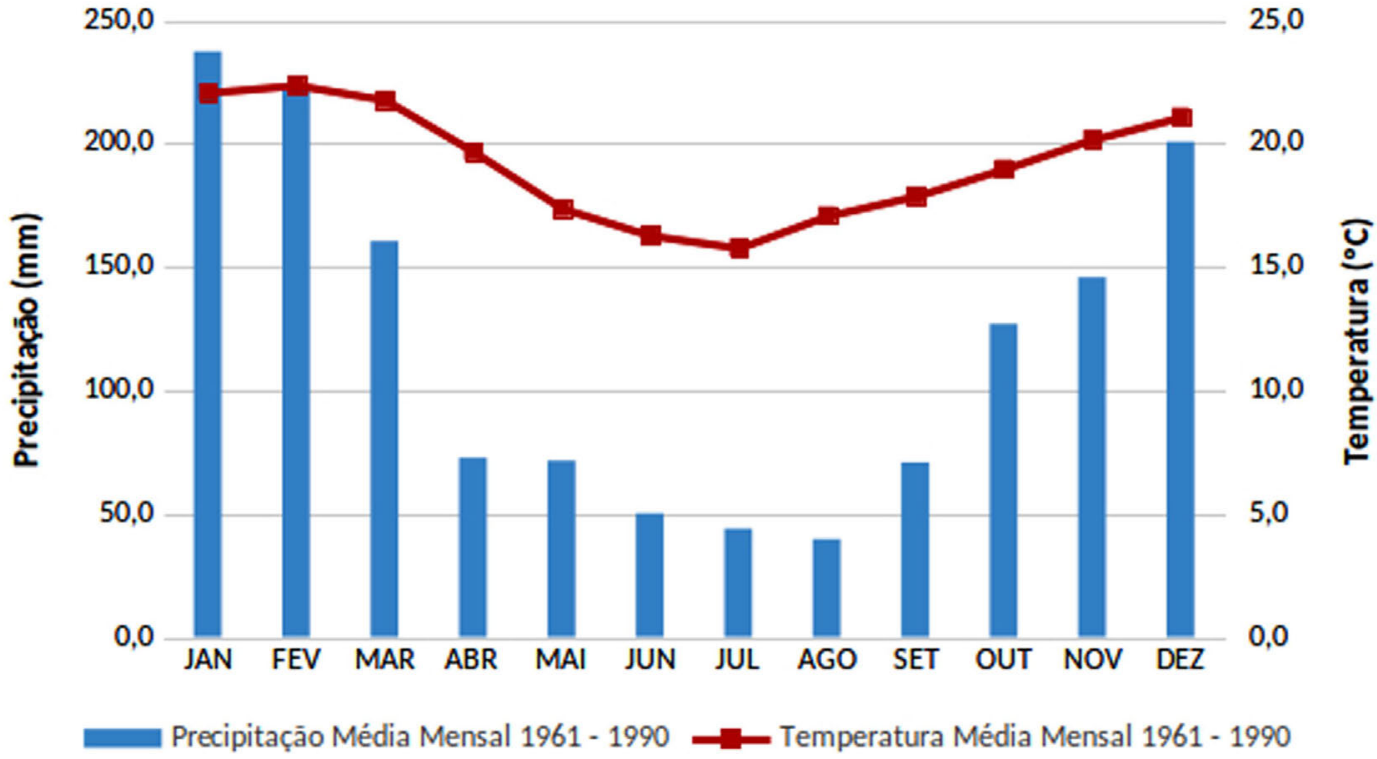

Figura 2 - Climograma de São Paulo-SP, dados da Estação Meteorológica Mirante de Santana (INMET). 


\subsubsection{Imagens utilizadas e georreferenciamento}

As imagens utilizadas nesta pesquisa são provenientes do satélite LANDSAT-5, sensor Thematic Mapper (TM), que possui 7 bandas espectrais, sendo três bandas na faixa do visível (bandas 1, 2 e 3), uma na faixa do infravermelho próximo (banda 4), duas na faixa do infravermelho médio (bandas 5 e 7) e uma na faixa do infravermelho termal (banda 6), tendo resolução espacial de $120 \mathrm{~m}$ para a banda 6 e $30 \mathrm{~m}$ para a demais bandas, sua resolução temporal é de 16 dias, com resolução radiométrica de 8 bits.

Foi utilizado neste trabalho o georreferenciamento denominado imagem a imagem, para tal procedimento foram utilizadas imagens Geocover, tais imagens são mosaicos feitos a partir de cenas do satélite LANDSAT-7, obtidas pelo sensor Enhanced Thematic Mapper Plus $(\mathrm{ETM}+)$ e reamostradas para uma resolução espacial de 14,25 m, onde é garantida a precisão das coordenadas, para ser utilizada como imagem referência. As imagens Geocover podem ser obtidas através do Earth Explorer que é um banco de dados operado pelo U.S. Geological Survey que contém diversos dados de sensoriamento remoto.

O processo de georreferenciamento imagem a imagem, necessitada da identificação de feições conhecidas (pontos de controle) e identificáveis tanto na imagem referência (Geocover), como nas imagens com necessidade de correção geométrica (LANDSAT-5). Neste trabalho foram utilizadas 4 cenas do satélite LANDSAT-5, em cada imagem foram selecionados 15 pontos de controle, tendo escolhido para o procedimento o polinômio de primeiro grau.

Todo o processamento necessário para a correção geométrica utilizou-se do software livre e de código aberto SPRING, em sua versão 5.2.5, rodando em ambiente operacional também livre UBUNTU, em sua versão 12.04 . Como passo inicial foi necessário a reamostragem da banda 6, para que a mesma fique com a resolução espacial de $30 \mathrm{~m}$, assim com as demais bandas. Nesta etapa foi desenvolvido um algoritmo utilizando a Linguagem Espacial para Geoprocessamento Algébrico (LEGAL), linguagem esta que está contido dentro do ambiente do SPRING, para que a imagem fosse reamostrada de forma correta. Posteriormente todas as bandas passaram pela conversão do formato nativo *.tiff, para o formato padrão do SPRING *.spg, está conversão é realizada em um software secundário que acompanha a instalação do SPRING, o IMPIMA. Dando sequência ao processo as imagens foram importadas para o SPRING onde foram determinados os pontos de controle e realizada a correção geométricas de todas as cenas, tomando como base a projeção UTM e o datum WGS84, obtendo erros Root Mean Squared Error (RMS) abaixo de 0,5, conforme Tabela 1, como é recomentado para uma correção geométrica eficiente.
Tabela 1 - Erros RMS depois da correção geométrica.

\begin{tabular}{cccc}
\hline Ano & Órbita & Ponto & Erro-RMS \\
\hline 1992 & 219 & 76 & 0,0083 \\
& & 77 & 0,227 \\
2006 & 219 & 76 & 0,156 \\
& & 77 & 0,065 \\
\hline
\end{tabular}

\subsubsection{Modelo digital de elevação (MIDE)}

Para esta pesquisa foi utilizado o algoritmo Surface Energy Balance Algorithm for Land (SEBAL), desenvolvido por Bastiaanssen (1995) e implementado em linguagem LEGAL por Cavalcante (2013), para se chegar a temperatura da superfície, que é umas das etapas desse algoritmo. O SEBAL, em seus primórdios, foi pensado para ser utilizado em uma área de relevo plano, entretanto, algumas pesquisas (Pace, 2004; Silva et al., 2005) introduzem o MDE nas etapas do SEBAL. Nesta pesquisa o modelo utilizado foi extraído através de imagens Topodata, que foi um projeto desenvolvimento pelo INPE, onde houve o melhoramento das imagens da missão Shuttle Radar Topography Mission (SRTM), preenchendo falhas e refinando a resolução espacial de 3 arcos-segundos (SRTM) para 1 arco-segundo (Topodata).

Com o mosaico feito através das imagens topodata, foi gerado o MDE da cidade de São Paulo, através do fatiamento de uma grade regular e representando o relevo por curvas de nível, reduzidas ao nível do mar, conforme (Fig. 3a), o MDE foi recortado utilizando o shapefile do IBGE. Como é perceptível no mapa de hipsometria, a cidade de São Paulo encontra-se quase que em sua totalidade na faixa de 752 a $792 \mathrm{~m}$ de altitude, possuindo elevações mais acentuadas ao norte da cidade, onde se localiza a Serra da Cantareira e mais baixas em uma pequena porção ao sul. Foram gerados dois perfis longitudinais que podem ser vistos nas Fig. $3(b, c)$ onde foram realizados cortes denominados A - A', que corta a cidade de São Paulo de Leste para Oeste e o corte denominado B - B', que corta a cidade de Norte a Sul, por último foi gerado o MDE e sobreposto a ele o mapa hipsométrico, conforme (Fig. 3d).

Outro ponto importante quando se está caracterizando o relevo é analisar a declividade, que pode ser entendida como a divisão da diferença de altura entre dois pontos e a distância entre os mesmos. Na Cidade de São Paulo, conforme Fig. 4, que segue a classificação da Solos (1999), existindo áreas classificadas como montanhosas, em uma porção ao Sul e na Serra da Cantareira ao Norte. A máxima declividade da cidade encontra-se em $61 \%$ e a mínima em 0,00004\%, com média de $6,01 \%$, conforme Tabela 2 , pode-se verificar que pela média, a classificação está sua maioria na faixa de suave ondulado. 

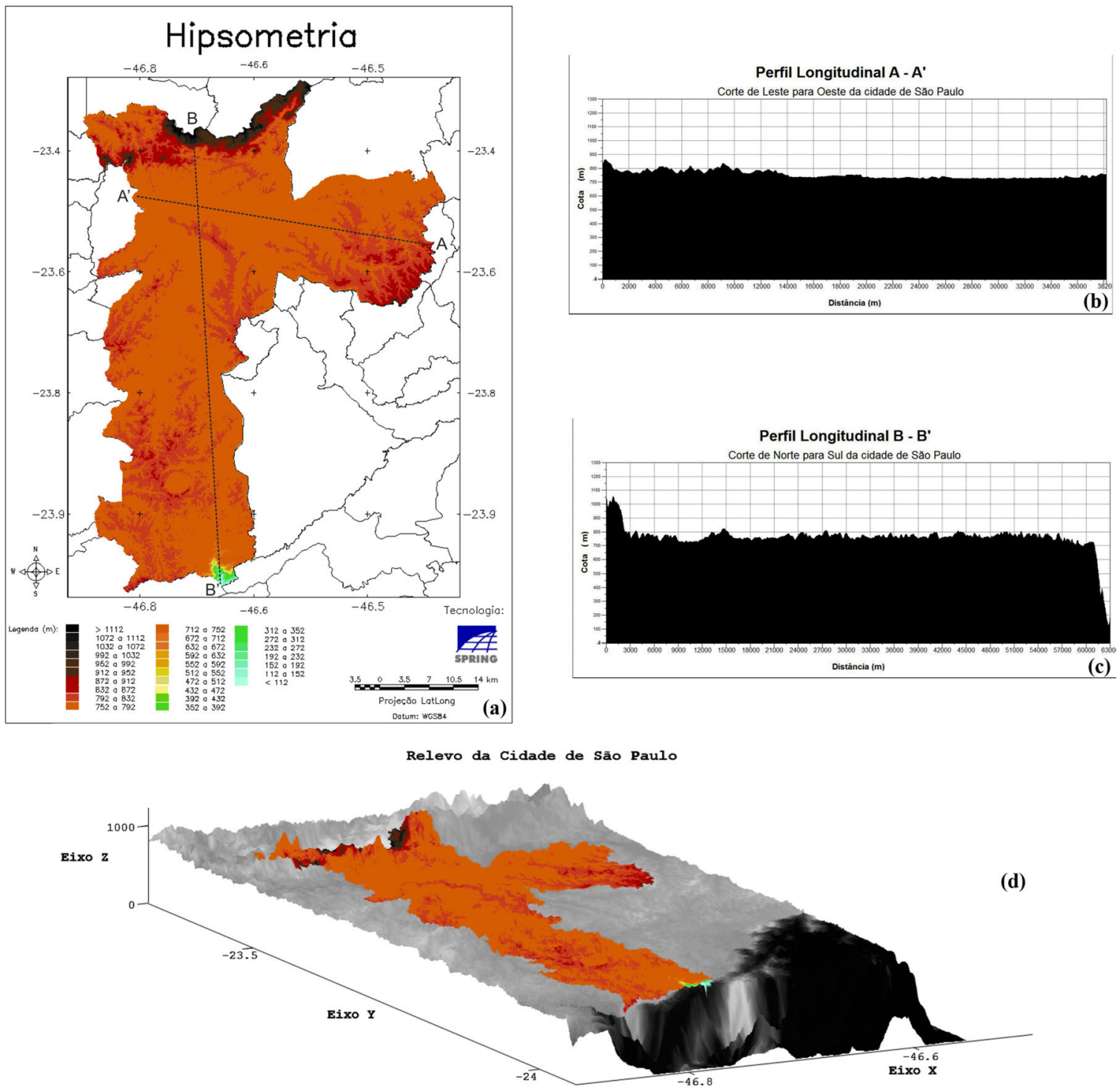

Figura 3 - Mapa hipsométrico da cidade de São Paulo.

\subsubsection{Processamento das variáveis}

O cálculo dos componentes necessários para a estimativa da temperatura da superfície e demais variáveis necessárias ao entendimento da formação de ilhas de calor, tais como: NDVI e albedo, foi processado através do SPRING, se utilizando do algoritmo implementado em LEGAL por Cavalcante (2013), todos os mapas e cartas gerados nesta pesquisa, foram produzidos através do módulo SCARTA, presente na interface do SPRING. A Fig. 5 mostra o fluxograma metodológico dos procedimentos realizados para o cálculo da temperatura da superfície.

\subsubsection{Calibração radiométrica das imagens Landsat-5 $\left(\mathrm{L}_{\lambda}\right)$}

A calibração radiométrica, trata da conversão dos números digitais $(N D)$, presentes das imagens brutas do
Landsat 5, em radiância espectral, que pode ser entendida como a energia proveniente do Sol, e que é refletida por cada pixel em uma unidade de área, em determinado tempo e em um determinado ângulo. Para o cálculo da radiância foi utilizado a fórmula proposta por Markham e Baker (1987), conforme (Eq. (1)).

$$
L_{\lambda(x, y)}=L_{\text {mim }}+\frac{L_{\text {max }}-L_{\text {min }}}{255} N D_{\lambda(x, y)}
$$

onde Lmin e Lmax são as radiâncias espectrais mínimas e máximas, onde a unidade de medida adotada é o W.m ${ }^{-2}$. $\mathrm{sr}^{-1} \cdot \mu \mathrm{m}^{-1}$, conforme Tabela 3 .

Para a conclusão da calibração radiométrica, é necessário a conversão das imagens de radiância espectral para reflectância espectral que segundo (Bastiaanssen et al., 1998; Allen et al., 2002; Silva et al., 2011), trata-se da 


\section{Declividade}

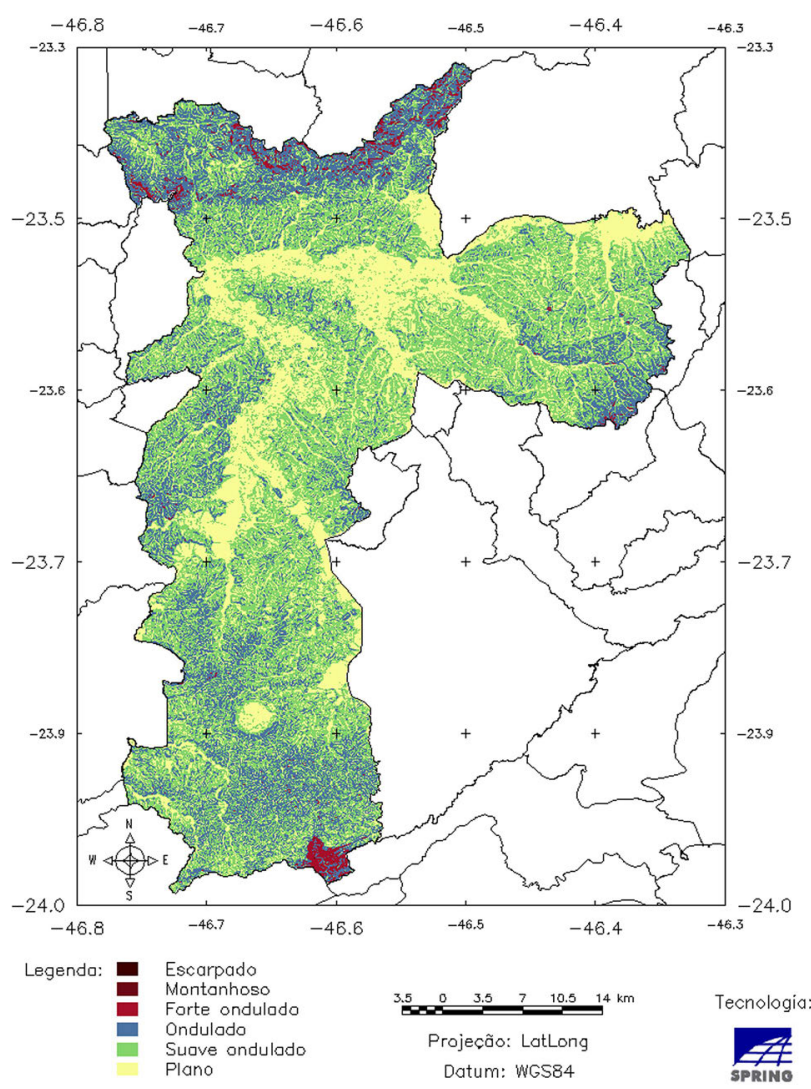

Figura 4 - Mapa hipsométrico da cidade de São Paulo.

Tabela 2 - Classificação da declividade segundo a faixa.

\begin{tabular}{lc}
\hline Classes & Faixa $(\%)$ \\
\hline Plano & 0 a 3 \\
Suave ondulado & 3 a 8 \\
Ondulado & 8 a 20 \\
Forte ondulado & 20 a 45 \\
Montanhoso & 45 a 75 \\
Escarpa & $>75$ \\
\hline
\end{tabular}

razão entre o fluxo de radiação solar emitido e o incidente, e pode ser expresso conforme a (Eq. (2)).

$$
\rho_{\lambda(x, y)}=\frac{\pi * L_{\lambda(x, y)}}{E S U N_{\lambda} * \cos \theta * d r}
$$

onde $\operatorname{ESUN\lambda }$, sendo a irradiância solar espectral de cada banda medido no topo da atmosfera, tendo com unidade de medida W.m ${ }^{-2} \cdot \mu \mathrm{m}^{-1}$, conforme Tabela $3, \theta$ é o ângulo solar zenital, $L_{\lambda(x, y)}$ é a radiância espectral calculado através da fórmula anterior e $d r$ é o inverso do quadrado da distância Terra-Sol, medido em unidades astronômicas.
2.3.4.2. Cálculo do albedo no topo da atmosfera $\left(\alpha_{T O A}\right)$ e do albedo da superfície $\left(\alpha_{(x, y)}\right)$

$\mathrm{O}$ albedo da atmosfera $\left(\alpha_{T O A}\right)$, nada mais é que o albedo sem correção, calculado através do somatório das reflectâncias espectrais multiplicados a respectivos pesos, conforme (Eq. (3)), em contra partida o albedo da superfície $\left(\alpha_{(x, y)}\right)$, é corrigido levando em consideração os efeitos da atmosfera, conforme (Eq. (4)).

$$
\begin{aligned}
\alpha_{T O A(x, y)} & =\sum_{\lambda=1}^{\eta}\left(\varpi_{\lambda} * \rho_{\lambda(x, y)}\right) \\
\rho_{\lambda(x, y)} & =\frac{E S U N_{\lambda}}{\sum_{E S U N_{\lambda}}} \\
\rho_{\lambda(x, y)} & =\frac{\alpha_{T O A(x, y)}-\alpha_{\rho a t h_{\text {rad }}}}{\tau^{2}}
\end{aligned}
$$

onde $\rho_{\lambda(x, y)}$ corresponde as reflectâncias espectrais referentes as bandas do satélite LANDSAT5, com exceção da banda 6, o $\alpha_{\text {pathrad }}$ é a radiação solar refletida pela atmosfera, tal valor segundo Bastiaansesen (1998), é correspondente a 0,03 e $\tau^{2}$ é a transmitância atmosférica, expressa pela (Eq. (5)), onde $o Z_{\text {médio }}$ é a altitude média da área de estudo, que neste trabalho foi encontrado pela média do $M D E$ já apresentado anteriormente.

$$
\tau=0,75+2 * 10^{-5} * z_{\text {médio }}
$$

2.3.4.3. Cálculo do Índice de Vegetação por Diferença Normalizada (NDVI)

O NDVI é segundo Tucker (1979) expresso pela razão entra a subtração da reflectância espectral das bandas do infravermelho próximo, e do vermelho, onde no LANDSAT 5 são representadas pelas bandas 4 e 3, respectivamente, conforme (Eq. (6)).

$$
N D V I=\frac{\rho 4_{(x, y)}-\rho 3_{(x, y)}}{\rho 4_{(x, y)}+\rho 3_{(x, y)}}
$$

onde $\rho 4_{(x, y)}$ e $\rho 3_{(x, y)}$ são as reflectâncias das bandas 3 e 4 . O NDVI é um índice que tem como princípio estimar o verdor da vegetação, tendo sua variação entre -1 a 1 , valores abaixo de zero, correspondem a corpos d'água, e quanto mais próximo de 1 mais verdor.

\subsubsection{Cálculo do Índice de Vegetação Ajustado ao Solo (SAVI)}

O SAVI é determinado conforme a expressão proposta por Huete (1988), conforme (Eq. (7)).

$$
S A V I_{(x, y)}=(1+L) \frac{\rho 4_{(x, y)}-\rho 3_{(x, y)}}{\rho 4_{(x, y)}+\rho 3_{(x, y)}}
$$

onde $L$ é uma constante de ajustamento que pela literatura, é comumente utilizado o valor de 0,5 , segundo Nicacio (2008). 


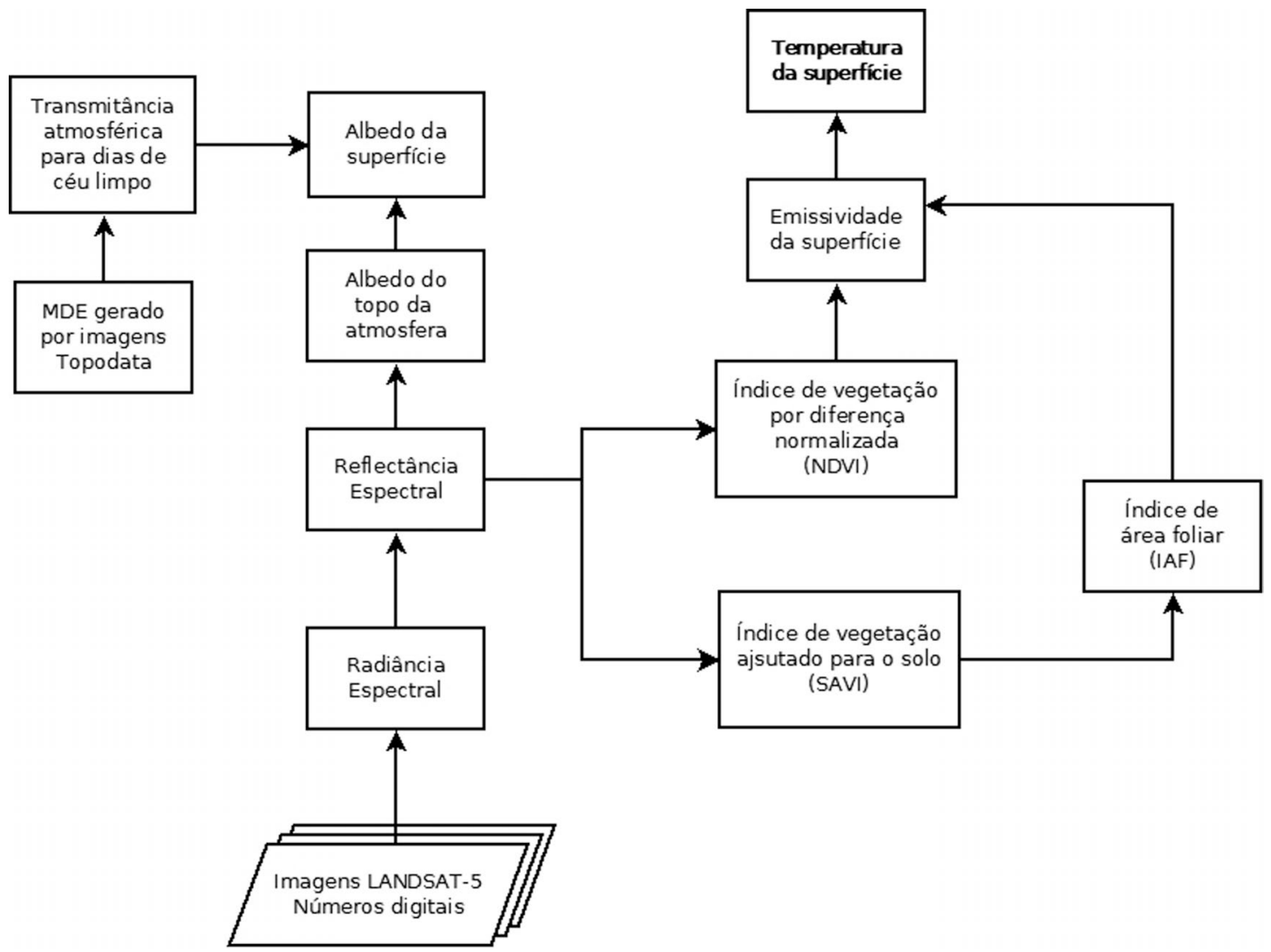

Figura 5 - Fluxograma metodológico do cálculo dos componentes do SEBAL. adaptado de Nicacio (2008).

Tabela 3 - Tabela com valores das radiâncias mínimas e máximas e da irradiância solar espectral no topo da atmosfera.

\begin{tabular}{|c|c|c|c|c|c|c|c|}
\hline \multirow{3}{*}{ Bandas espectrais } & \multicolumn{6}{|c|}{ Radiância espectral $\mathrm{L}_{\min }$ e $\mathrm{L}_{\max }$} & \multirow{3}{*}{$\begin{array}{l}\text { Irradiância solar no topo da atmosfera } \\
\qquad \text { w.m }{ }^{-2 *} \mu \mathrm{m}^{-1} \\
\end{array}$} \\
\hline & \multicolumn{2}{|c|}{$\begin{array}{l}\text { A partir de } 1 \text { de março de } 1984 \text {, } \\
\text { até } 4 \text { de maio de } 2003\end{array}$} & \multicolumn{2}{|c|}{$\begin{array}{l}\text { A partir de } 5 \text { de março de } \\
2003 \text {, até } 1 \text { de abril de } 2007\end{array}$} & \multicolumn{2}{|c|}{$\begin{array}{l}\text { A partir de } 2 \text { de abril de } \\
2007 \text {, até atualmente }\end{array}$} & \\
\hline & $\mathrm{L}_{\min }$ & $\mathrm{L}_{\max }$ & $\mathrm{L}_{\min }$ & $\mathrm{L}_{\max }$ & $\mathrm{L}_{\min }$ & $\mathrm{L}_{\max }$ & \\
\hline 1 & $-1,52$ & 152,10 & $-1,52$ & 193,0 & $-1,52$ & 193,0 & 1957 \\
\hline 2 & $-2,84$ & 296,81 & $-2,84$ & 365,0 & $-2,84$ & 365,0 & 1826 \\
\hline 3 & $-1,17$ & 204,30 & $-1,17$ & 264,0 & $-1,17$ & 264,0 & 1554 \\
\hline 4 & $-1,51$ & 206,20 & $-1,51$ & 221,0 & $-1,51$ & 221,0 & 1036 \\
\hline 5 & $-0,37$ & 27,19 & $-0,37$ & 30,2 & $-0,37$ & 30,2 & 215,0 \\
\hline 6 & 1,25 & 15,31 & 1,25 & 15,31 & 1,25 & 15,31 & - \\
\hline 7 & $-0,15$ & 14,38 & $-0,15$ & 16,5 & $-0,15$ & 16,5 & 80,67 \\
\hline
\end{tabular}

Fonte: Adaptado de Chander et al. (2007).

\subsubsection{Cálculo do Índice de Área Foliar (IAF)}

O $I A F$ é um índice que foi estabelecido para calcular a razão entre a área foliar total e a área ocupado pela cultura, segundo Allen et al. (2002), é expresso por uma formulação empírica, conforme (Eq. (8)).

$$
I A F=-\frac{\ln \left(\frac{0,69-I V A S}{0,59}\right)}{0,91}
$$

2.3.4.6. Cálculo das Emissividades $\left(\varepsilon_{\mathrm{NB}}\right.$ e $\varepsilon$ )
A $\varepsilon_{N B}$ é medida no domínio espectral contido na banda termal do sensor TM (banda 6) enquanto a $\varepsilon$ é medida na faixa da banda larga. Segundo Allen et al. (2002), ambas podem ser calculadas conforma as Eqs. (9) e (10), seguindo para isso a condição que $N D V I>0$ e $I A F<3$.

$$
\begin{gathered}
\varepsilon N B_{(x, y)}=0,97+0,0033 * I A F_{(x, y)} \\
\varepsilon_{(x, y)}=0,95+0,01 * I A F_{(x, y)}
\end{gathered}
$$


Considerando condições onde $I A F \geq$, então $\varepsilon N B_{(x, y)}=\varepsilon_{(x, y)}$ $=0,98$ e $N D V I<0, \log 0 \varepsilon N B_{(x, y)}=0,99$ e $\varepsilon_{(x, y)}=0,985$, segundo Allen et al. (2002).

\subsubsection{Cálculo da Temperatura da Superfície (TS)}

A $T s$ calculada por sensoriamento remoto orbital é determinada de forma instantânea, ou seja, é a temperatura referente ao dia e horário da passagem do sensor imageador sobre a superfície, é determinada levando em consideração a radiância espectral da banda termal (banda 6) do satélite LANDSAT5, sensor TM e a $\varepsilon_{N B}(x, y)$, sendo seus valores em dados em graus Kelvin $(K)$ e sua formulação é encontrada, conforme a (Eq. (11)).

$$
T s=\frac{K_{2}}{\ln \left(\frac{\varepsilon_{N B} K_{1}}{L_{\lambda, 6}}+1\right)}
$$

sendo, $K_{1}$ e $K_{2}$ são constantes que equivalem a $607,76 \mathrm{~W} \cdot \mathrm{m}^{-2} \cdot \mathrm{sr}^{-1} \cdot \mu \mathrm{m}^{-1}$ e $1260,56 \mathrm{~K}$, respectivamente.

Com o intuito de deixar os dados de temperatura com melhor compreensão, e tendo em vista que no Brasil a unidade de medida adotada para temperatura é graus Celsius $\left({ }^{\circ} \mathrm{C}\right)$, a imagem de temperatura da superfície foi convertida para graus Celsius através da subtração de todos os pixels da imagem pelo valor 273 .

\subsubsection{Mapa de uso e cobertura do solo}

Além das análises das variáveis apresentadas no capítulo anterior, nesta pesquisa foi gerado um mapa de uso e cobertura do solo, através da classificação das imagens do LANDSAT 5, a imagem escolhida para a classificação foi a referente ao ano de 2006, por ser a mais recentes das duas escolhidas nesta pesquisa, demostrando assim a realidade das características de uso e cobertura na área de estudo, tais imagens foram classificadas utilizando-se o método de classificação supervisionada, tal método tem por objetivo se utilizar do conhecimento do usuário para "treinar" o software a distinguir as classes por ele escolhidas, neste estudo especificamente foram escolhidas quatro classes, sendo elas: solo exposto, vegetação (arbórea, herbácea, gramínea, dentre outras), água e área urbana. Segundo Liotte (2000), para que a classificação seja mais precisa é utilizado nesta distinção a chamada Verdade Terrestre (Ground Truthing), termo utilizado no sensoriamento remoto para nomear o conhecimento prévio do usuário sobre a área a ser classificada. Para que a amostra de treinamento seja considerada estatisticamente confiável, são necessários de 10 a 100 pixels selecionados por classe, levando-se em consideração uma amostra homogenia, entretanto, que contenha toda a variabilidade existe dentre desta amostra (Crósta, 1992; Fonseca, 1988). Vale ressaltar, que o número de pixels necessários para se obter precisão aumenta à medida que a variabilidade entre classe é ampliada.
O classificador escolhido nesta pesquisa foi $\mathrm{o}$ Máxima Verossimilhança (MAXVER), pois é o classificador mais amplamente utilizado em métodos de classificação supervisionada. O classificador MAXVER considera a ponderação das distâncias entre as médias dos níveis digitais (ND) das classes, para este processo utiliza bases estatísticas relativamente complexas. Segundo Richards (1999), o método Máxima Verossimilhança parte do pressuposto que a classificação errônea de um pixel não tem maior significância do que a classificação errônea de qualquer outro pixel presente na imagem.

Posteriormente ao processo de classificação, é necessário se calcular a exatidão alcançada no mapa de uso e cobertura do solo gerado, esta exatidão parte do princípio, que uma determinada medida tem proximidade com seu valor real, partindo deste contexto, é necessário proceder com cálculos estatísticos, analisando o produto classificado, para que a acurácia da classificação seja obtida Bernardes (2006).

Esta avaliação pode ser obtida por meio de coeficientes de concordância da matriz de confusão, segundo Vieira (2000), é através desta matriz que se pode derivar medidas e por consequência verificar erros oriundos do processo de classificação. O coeficiente mais comumente utilizado é o KAPPA, que segundo Congalton (1991), é satisfatório na avaliação da precisão de uma classificação, pelo fato de analisar toda a matriz de confusão, sem se ater somente aos elementos da diagonal principal, como ocorre na exatidão global.

O software livre e de código aberto SPRING, utilizado em todos os processos desta pesquisa, em seu relatório estatístico apresenta entre outros índices, o TAU, proposto por Redmond (1995), na tentativa de corrigir a deficiência do KAPPA, que por incluir em seus cálculos a concordância real da amostra, poderia superestimar o grau de concordância e subestimar a precisão. Figueiredo (2007) em estudo de comparação de coeficientes mostra que a diferença entre o KAPPA e o TAU é tão pequena, que ambos podem ser utilizados em avaliação de acurácia de classificação, sem que haja prejuízo para os estudos.

Para sanar qualquer tipo de dúvida com relação aos índices de análise de classificação, e sobre a exatidão alcançada no mapa de uso e cobertura do solo desta pesquisa, foi elaborada uma planilha no formato *.ods seguindo a metodologia de cálculo do índice KAPPA, presente em Figueiredo (2007), e comparada a análise dada através do índice TAU do SPRING. Para o índice TAU foi alcançada uma exatidão de $99,9342 \%$, enquanto para o índice KAPPA foi de 99,9442\%, segundo Fonseca (2000) se o índice KAPPA encontra-se entre 80 e $100 \%$ a classificação é considerada excelente. 


\section{Resultados e Discussão}

Visando analisar a correlação de temperatura da superfície com uso e cobertura do solo, na Fig. 6 é apresentado o mapa gerado para o ano de 2006, visando identificar alvos que representem maior variação de aumento da temperatura, assim como identificar a que tipo de uso estas variações estão ligadas. Como esperado o município de São Paulo possui a maior parte do território ocupada por área urbana, possuindo o Parque Estadual da Cantareira ao Norte e o Parque Estadual da Serra do Mar ao Sul, como suas maiores reservas de mata. $\mathrm{E}$ dois grandes corpos d'água, sendo a mais a Oeste a Represa de Guarapiranga e a Leste a Represa Billings.

As Figs. 7-8 mostram os valores obtidos para a Ts, nas datas de 26/09/1992 e 14/09/2006 e os valores estimados de NDVI, respectivamente. Para a temperatura referente ao ano de 1992, a temperatura mínima alcançada foi de $16,8{ }^{\circ} \mathrm{C}$, tendo uma máxima de $37,0{ }^{\circ} \mathrm{C}$ e média de $26,58{ }^{\circ} \mathrm{C}$, observando o mapa de temperatura gerado é perceptível que as menores temperaturas, estão localizadas em corpos d'água alcançando temperaturas inferiores a $23,0{ }^{\circ} \mathrm{C}$, condição similar é encontrada no extremo norte de São Paulo, região pertencente a Serra da Cantareira; no

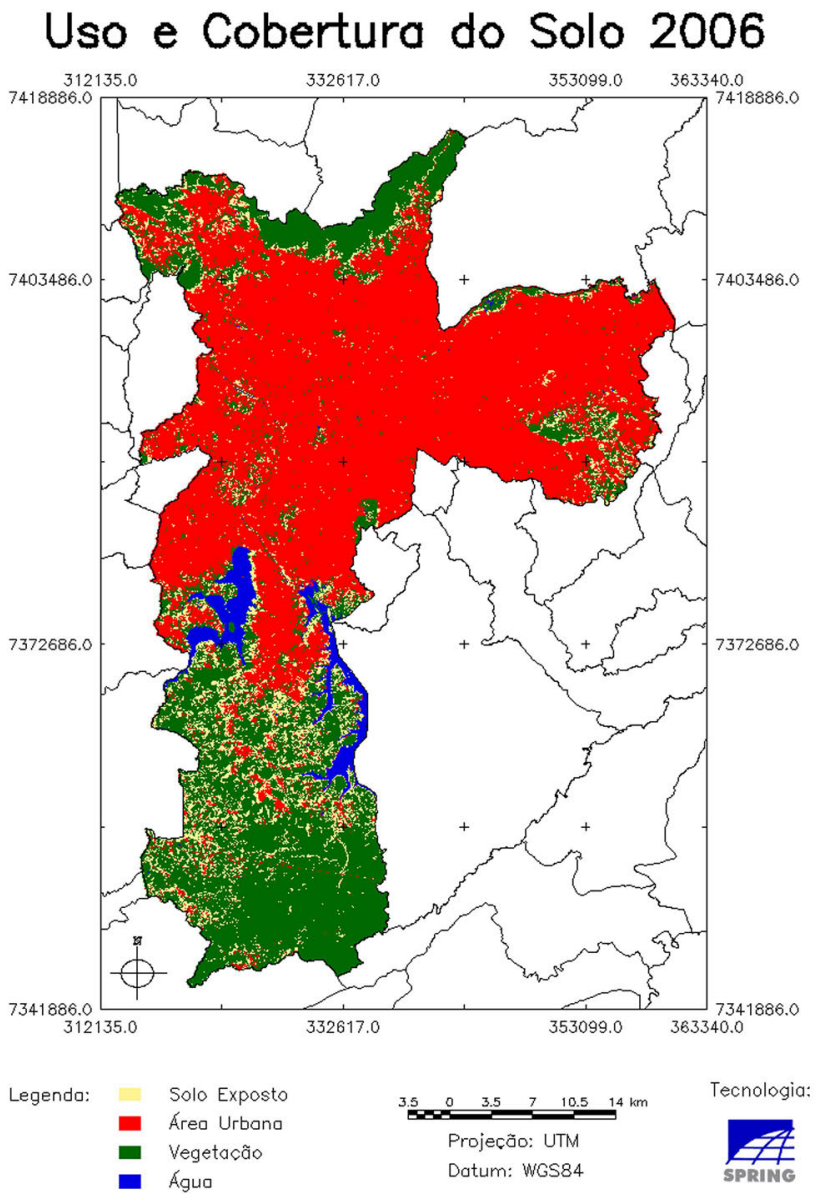

Figura 6 - Mapa de uso e ocupação do solo (2006).
Sul da cidade está localizada uma região com bastante área vegetada, onde a temperatura alcançou valores entre 23,0 e $28,0{ }^{\circ} \mathrm{C}$; na área urbana da cidade as temperaturas já chegam a variações de 30,0 a $34,0{ }^{\circ} \mathrm{C}$, tendo pequenas manchas no centro da área urbana de São Paulo, com valores de 34,0 a $36,0{ }^{\circ} \mathrm{C}$.

Analisando o mapa de temperatura da superfície para o ano de 2006, a temperatura alcançou mínima de $10,8{ }^{\circ} \mathrm{C}$ e máxima de $42,9{ }^{\circ} \mathrm{C}$, com média de $28,8{ }^{\circ} \mathrm{C}$, igualmente ao ano de 1992 as menores temperaturas estão localizadas em corpos d'água, e em algumas regiões da Serra da Cantareira. A região urbana de São Paulo teve um aumento da temperatura, subindo a variação para 34,0 a $38,0^{\circ} \mathrm{C}$, com pequenas manchas no centro da área urbana alcançando valores superiores a $38^{\circ} \mathrm{C}$.

Houve um aumento da temperatura da superfície em toda área do município de São Paulo, porém, em maior proporção nas áreas localizadas em torno da Marginal Tietê, centro de São Paulo e Rodovia Ayrton Senna. O que pode ser explicado por serem zonas mais baixas, com pouca ou quase nenhuma vegetação, uma densidade de prédios muito alta; o que irradia mais calor e reduz a circulação dos ventos, além da presença de zonas industriais.

Analisando os pontos de variação de temperatura, fora as ilhas de calor, São Paulo apresenta zonas de temperatura baixas, como forma de identificação foram divididas 4 (quatro) áreas que podem ser vistas na (Fig. 9a), representando ilhas de frescor.

A área 1 está exatamente sobre o Jardim Zoológico de São Paulo, região com mais de $824.000 \mathrm{~m}^{2}$ de reserva de Mata Atlântica, o que faz com que as temperaturas de superfície fiquem entre 23 e $28{ }^{\circ} \mathrm{C}$. A área 2 é onde está localizado o Parque do Carmo, apesar de estar próximo a áreas industriais com temperaturas acima dos $34{ }^{\circ} \mathrm{C}$, o parque concentra variações de temperatura entre 23 e $30{ }^{\circ} \mathrm{C}$, em uma área de aproximadamente $1.500 .000 \mathrm{~m}^{2}$. A área 3 representa uma das áreas de maior disparidade socioeconômica, possuindo bairros de alta renda como o Panamby, que possuem grandes áreas verdes, ao mesmo tempo que possui um dos maiores aglomerados subnormais (área 3.1), Paraisópolis, representando uma ilha de alta temperatura no meio da região arbórea. Finalizando a análise foi gerado um mapa diferença entre os anos de 2006 e 1992, toda a área em vermelho sofreu uma ampliação da temperatura da superfície, todas as áreas em branco uma redução, neste caso somente os corpos d'água (Fig. 9b).

\section{Considerações Finais}

O município de São Paulo vem apresentando uma variação crescente da temperatura da superfície, sendo em sua grande maioria resultado do crescimento urbano desordenado e da impermeabilização do solo através de revestimento asfáltico. 

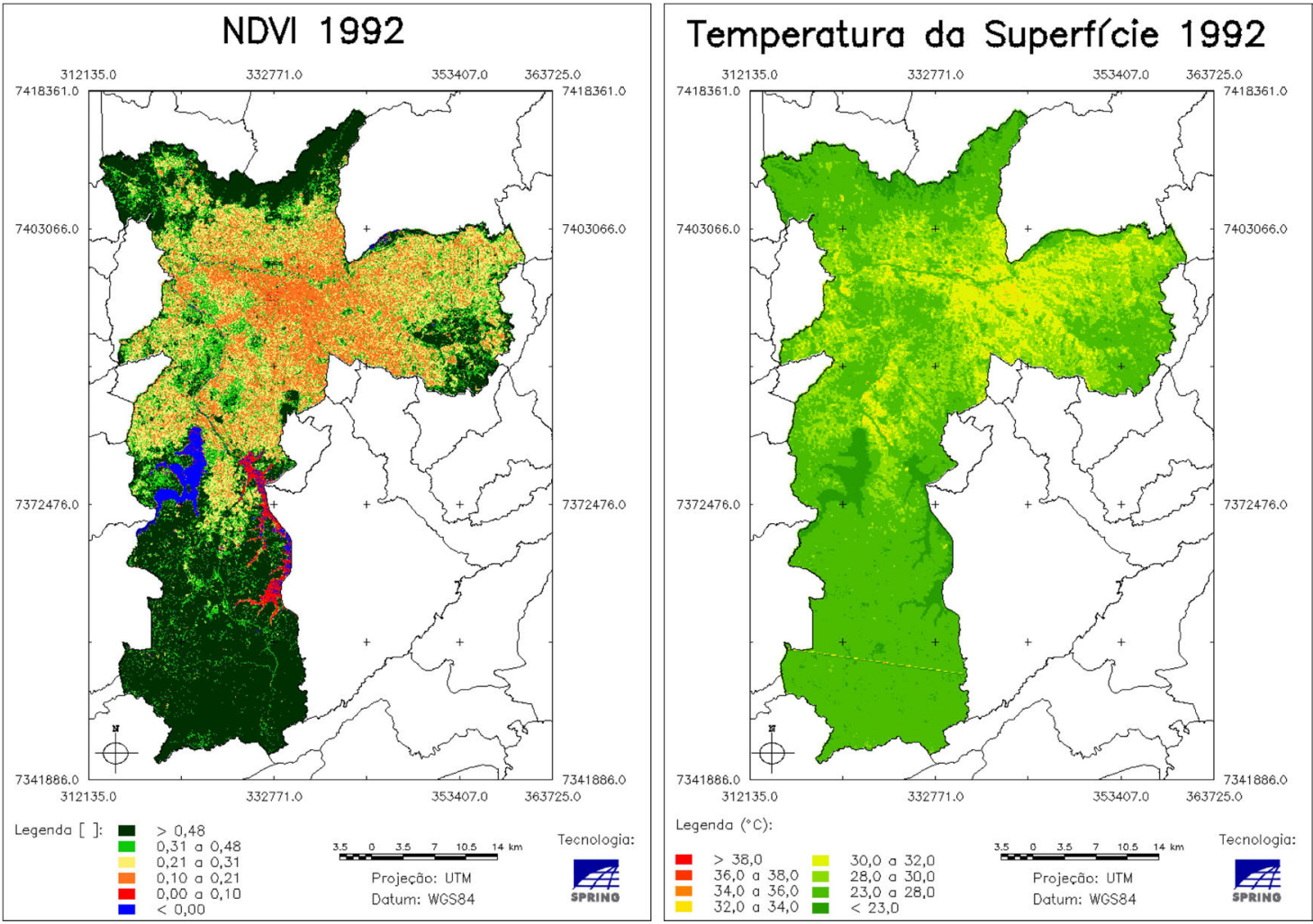

Figura 7 - NDVI (a) e Temperatura da superfície (b) para o ano de 1992.
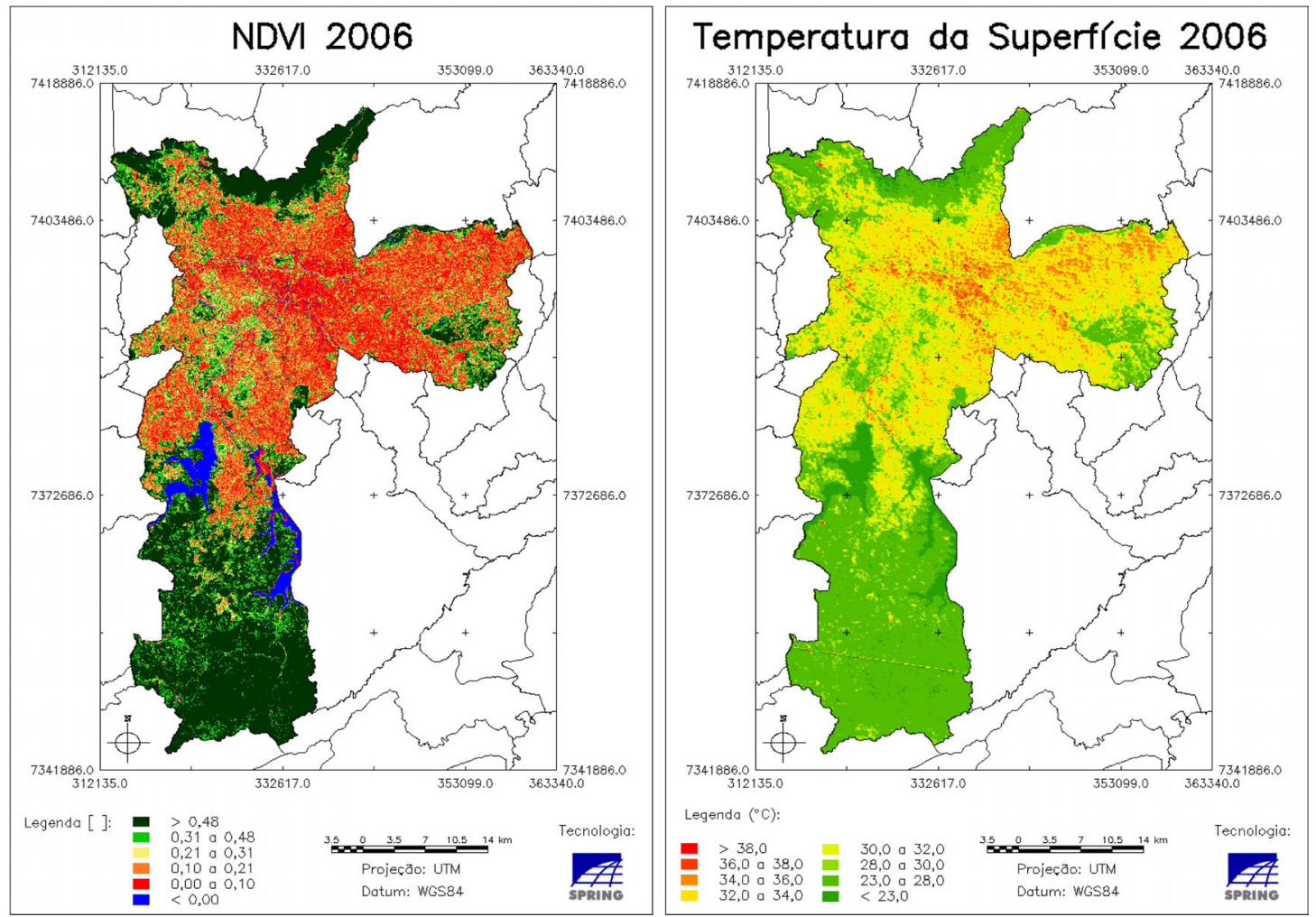

Figura 8 - NDVI (a) e Temperatura da superfície (b) para o ano de 2006. 

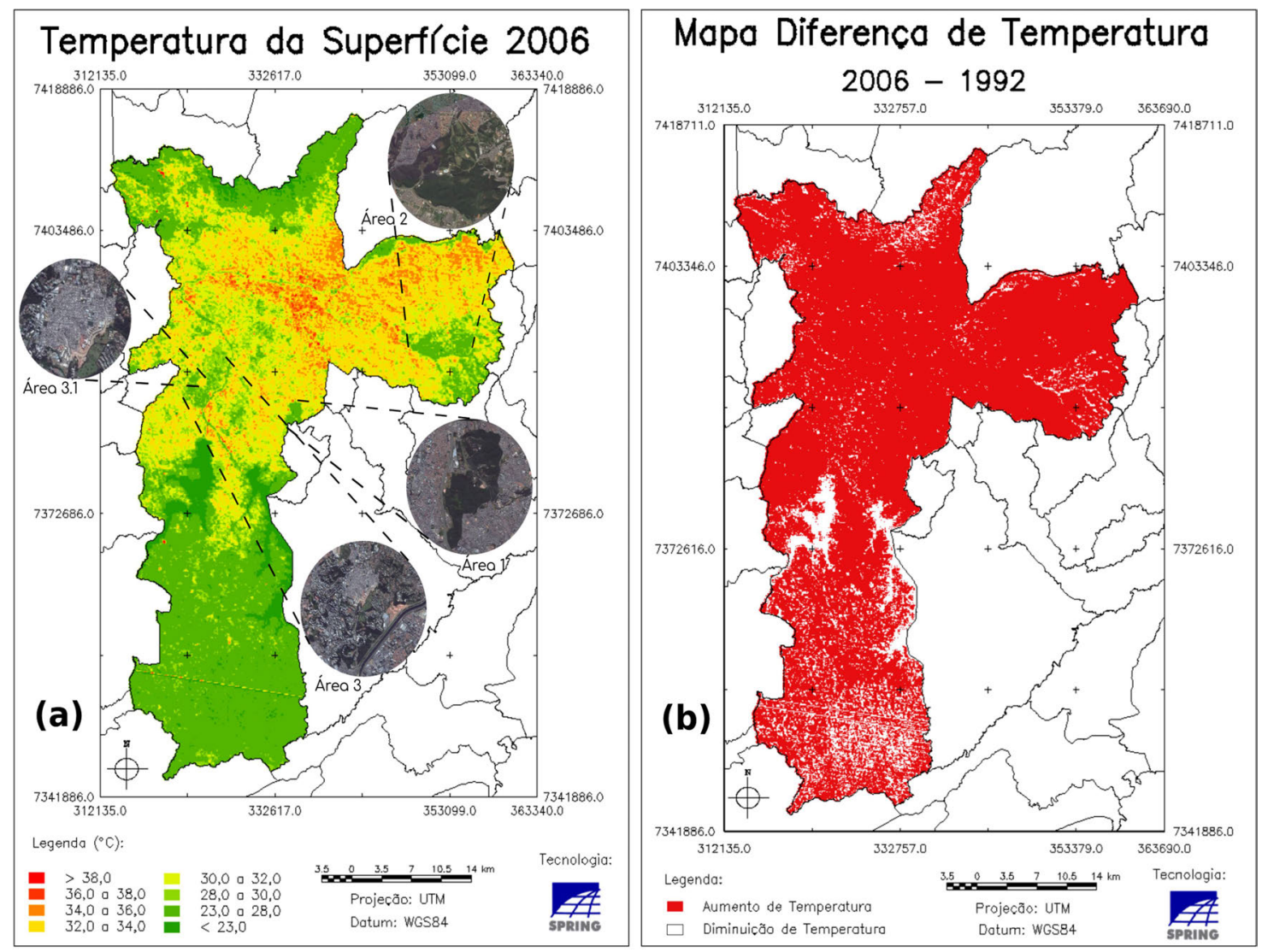

Figura 9 - (a) Comparações de áreas para o ano de 2006, (b) Mapa diferença 2006-1992.

A presença de grandes zonas industriais o que apresenta uma grande concentração de telhados de zinco, amianto ou alumínio, ensejando uma maior concentração de calor, também são fatores que devem ser considerados para a formação das ilhas de calor.

As imagens do Landsat 5 apesar de uma resolução espacial de $30 \mathrm{~m}$, foram suficientes para apresentar resultados significantes para descriminação de áreas de ilhas de calor e zonas de frescor com as apresentadas.

Fica evidente que ter a preocupação com a arborização da cidade tem uma total relação com a melhor condição de vida da sociedade, as árvores como visto, influenciam de forma direta na temperatura do ambiente, alteração na variação térmica, além de melhorar a questão da poluição do ar.

\section{Referências}

ALLEN, R.G.; TASUMI, M.; TREZZA, R.; WATERS, R.; BASTIAANSSEN, W. et al. Surface Energy Balance Algorithms for Land - SEBAL. Advance Training and User's Manual-Idaho Implementation, version, v. 1, p. 97, 2002. BASTIAANSSEN, W.G.M. Regionalization of Surface Flux Densities and Moisture Indicators in Composite Ter- rain: A Remote Sensing Approach Under Clear Skies in Mediterranean Climates. Wageningen: SC-DLO, 1995.

BASTIAANSSEN, W.G.; MENENTI, M.; FEDDES, R.A.; HOLTSLAG, A.A.M. et al. A remote sensing surface energy balance algorithm for land (SEBAL). 1. Formulation. Journal of Hydrology, v. 212, p. 198-212, 1998.

BERNARDES, T. Caracterização do Ambiente Agrícola do Complexo Serra Negra por meio de Sensoriamento Remoto e Sistemas de Informação Geográfica. Dissertação de Mestrado, Lavras, UFLA, p. 119, 2006.

CAVALCANTE, L.B. Utilização de Software Livre SPRING e Sensores Orbitais Para o Cálculo do Saldo de Radiação da Superfície na Região do Submédio São Francisco. Monografia, Graduação em Engenharia de Agrimensura, Instituto de Geografia, Desenvolvimento e Meio Ambiente, Universidade Federal de Alagoas, 2013.

CHANDER, GYANESH; MARKHAM; BRIAN L.; BARSI, JULIA A. et al. Revised Landsat-5 thematic mapper radiometric calibration. IEEE Geoscience and Remote Sensing Letters, v. 4, n. 3, p. 490-494, 2007.

CONGALTON; R.G. A review of assessing the accuracy of classifications of remotely sensed data. Remote Sensing of Environment, v. 37, n. 1, p. 35-46, 1991.

CRÓSTA, A.P. Processamento Digital de Imagens de Sensoriamento Remoto. São Paulo, Tese de Doutorado. Instituto de Geociências, Universidade de Campinas. p. 173, 1992. 
FIGUEIREDO, G.C.; VIEIRA, C.A.O. Estudo do comportamento dos índices de Exatidão Global, Kappa e Tau, comumente usados para avaliar a classificação de imagens do sensoriamento remoto. Simpósio Brasileiro de Sensoriamento Remoto, v. 13, p. 5755-5762, 2007.

FONSECA, L.M.G. Restauração de Imagens do Satélite Landsat Por Meio de Técnicas de Projeto de Filtros FIR. Dissertação de Mestrado em Engenharia Eletrônica, Instituto Tecnológico de Aeronáutica, São José dos Campos, 1988.

FONSECA, L.M.G. Processamento Digital de Imagens. Tese de Doutorado em Computação Aplicada, Instituto Nacional de Pesquisas Espaciais. São José dos Campos. p. 105, 2000.

GARTLAND, L. Ilhas de Calor. São Paulo: Oficina de Textos, p. 9-10, 2010.

HUETE, A.R. A soil-adjusted vegetation index (SAVI). Remote Sensing of Environment, v. 25, n. 3, p. 295-309, 1988.

IBGE - INSTITUTO BRASILEIRO DE GEOGRAFIA E ESTATÍSTICA. Estimativa da População. Rio de Janeiro: IBGE, 2019.

JENSEN, J.R.; EPIPHANIO, J.C.N. Sensoriamento Remoto do Ambiente: Uma Perspectiva em Recursos Terrestres. São Paulo: Parêntese, 672 p., 2009.

KÖPPEN, W.Z.A.V.; Prof. GEEN, D.G. Schwankungen der Sonnenstrahlung seit 18000 Jahren. GeolRundsch. v. 19, p. 314-315, 1928.

LIOTTE, S.V. Utilização de Técnicas de Geoprocessamento Para Apoio ao Planejamento Físico-Territorial do Município de Pariquera-Açu/SP. Tese de Doutorado, Universidade de São Paulo, São Paulo, 2000.

NICÁCIO, R.M. Evapotranspiração Real e Umidade do Solo Usando Dados de Sensores Orbitais e a Metodologia SEBAL na Bacia do Rio São Francisco. Tese de Doutorado, UFRJ, Rio de Janeiro, 320 p., 2008.

PACE, F.T.D. Estimativa do Balanço de Radiação à Superfície Terrestre Utilizando Imagens TM-Landsat 5 e Modelo de Elevação Digital. Tese de Doutorado, DCA/CCT/ UFCG, Campina Grande. 2004.

MA, Z; REDMOND, R.L. Tau coefficients for accuracy assessment of classification of remote sensing data. Photogrammetric Engineering and Remote Sensing, v. 61, n. 4, p. 435-439, 1995.

RICHARDS, J.A. Remote Sensing Digital Image Analysis. Berlin: Springer, p. 10-38, 1999.

SILVA, B.B.; LOPES, G.M.; AZEVEDO, P.V. Balanço de radiação em áreas irrigadas utilizando imagens Landsat 5-TM. Revista Brasileira de Meteorologia, v. 20, n. 2, p. 243252, 2005.

SILVA, B.B.; BRAGA, A.C.; BRAGA, C.C. Balanço de radiação no perímetro irrigado São Gonçalo-PB mediante imagens orbitais. Revista Caatinga, v. 24, n. 3, p. 145-152, 2011.

EMBRAPA SOLOS. Sistema Brasileiro de Classificação de Solos. Brasília: Embrapa Solos, 1999.

TUCKER, C.J. Red and photographic infrared linear combinations for monitoring vegetation. Remote Sensing of Environment. v. 8, n. 2, p. 127-150, 1979.

VALERIANO, M.M. Modelo digital de variáveis morfométricas com dados SRTM para o território nacional: o projeto TOPODATA. In: XII Simpósio Brasileiro de Sensoriamento Remoto. São José dos Campos: INPE, p. 1-8, 2005.

VALERIANO, M.M.; ROSSETTI, D.F.; ALBUQUERQUE, P.C.G. Topodata: desenvolvimento da primeira versão do banco de dados geomorfométricos locais em cobertura nacional. Simpósio Brasileiro de Sensoriamento Remoto, p. 1-8, 2009.

VIEIRA, S.R. Tópicos em Ciência do Solo. Viçosa: Sociedade Brasileira de Ciência do Solo, p. 1-53, 2000.

License information: This is an open-access article distributed under the terms of the Creative Commons Attribution License (type CC-BY), which permits unrestricted use, distribution and reproduction in any medium, provided the original article is properly cited. 